\title{
A Unique Immunofluorescence Protocol to Detect Protein Expression in Vascular Tissues: Tacking a Long Standing Pathological Hitch
}

\author{
Puneet GANDHI, Richa KHARE
}

Department of Research, Bhopal Memorial Hospital \& Research Centre, BHOPAL-38, INDIA

\begin{abstract}
Objective: Autofluorescence induced interference is one of the major drawbacks in immunofluorescence analysis of formalin-fixed paraffin-embedded tissues, as it decreases the signal-to-noise ratio of specific labeling. Apart from aldehyde-fixation induced artifacts; collagen and elastin, red blood cells and endogenous fluorescent pigment lipofuscin are prime sources of autofluorescence in vascular and aging tissues. We describe herein, an optimized indirect-immunofluorescence method for archival formalin-fixed paraffin-embedded tissues tissues and cryo sections, using a combination of 3-reagents in a specific order, to achieve optimal fluorescence signals and imaging.

Material and Method: Human telomerase reverse transcriptase, a protein implicated as a proliferation marker, was chosen relevant to its expression in solid tumors along with 3 other intracellular proteins exhibiting nuclear and/or cytoplasmic expression. Staining was performed on 10 glioma tissue sections along with 5 of their cryo sections, 5 sections each of hepatocellular, lung, papillary-thyroid and renal cell carcinoma, with 10 non-malignant brain tissue samples serving as control. Specimens were imaged using epifluorescence microscopy, followed by software-based quantification of fluorescence signals for statistical analysis and validation.

Results: We observed that the combined application of sodium-borohydride followed by crystal violet before antigen retrieval and a Sudan black B treatment after secondary antibody application proved to be most efficacious for masking autofluorescence/non-specific background in vascular tissues.

Conclusion: This unique trio-methodology provides quantifiable observations with maximized fluorescence signal intensity of the target protein for longer retention time of the signal even after prolonged storage. The results can be extrapolated to other human tissues for different protein targets.
\end{abstract}

Key Words: Autofluorescence, Immunofluorescence, Immunohistochemistry, Signal intensity

\section{INTRODUCTION}

Immunofluorescence (IF) is a sensitive and versatile tool for histological studies (1). However, IF staining of formalinfixed-paraffin-embedded (FFPE) tissues is not a routinely used method in clinical pathology due to the associated autofluorescence. On the other hand, immunoperoxidase staining of FFPE tissues is a fundamental technique for diagnostic pathology as well as research, although there are some major limitations associated with it. The first among these is the resolution of antigen localization by light microscopy that remains limited because chromogenic substrate saturates and precipitates easily. Another issue is the thickness of the sections that are imaged for analysis (2). Sometimes the antigen diffuses prior to fixation, making localization difficult specially in the case of soluble proteins like Glial Fibrilliary Acidic Protein (GFAP) (3). All of these conditions hinder evaluation. Additionally, the conventional staining method is less specific than antigen

(Turk Patoloji Derg 2018, 34:57-65)

Received : 21.03.2017 Accepted : 14.06.2017 localization by immunofluorescence, making quantifiable assessment difficult and therefore inherently subjective (4).

Autofluorescence induced interference is one of the major hitches in IF analysis of FFPE tissues, as it decreases the signal-to-noise ratio of specific labeling. The greatest source of autofluorescence in mammalian tissues is artifacts due to aldehyde fixation along with endogenous fluorophores: collagen, elastin, lipofuscin, red blood cells, and others. Vascular tissues contain massive amounts of collagen and elastin, along with haem, which are typical constituents of blood vessels (5-7), while lipofuscin is known to accumulate with aging and oxidative stress in various tissues $(8,9)$. Under a fluorescence or confocal microscope, lipofuscin appears as tiny, dot like structures within the cells, strongly fluorescing over a wide excitation range of 360-647 nm (10), which overlaps with the commonly used fluorophores and interferes with specific immuno-fluorescent signals (11). In the present method validation, we describe the optimized

Correspondence: Puneet GANDHI

Bhopal Memorial Hospital \& Research Centre,

Department of Research, BHOPAL, INDIA

E-mail: puneetgandhi67@yahoo.com Phone: +917552742152 
indirect IF method on vascular, archival FFPE tissues and cryo sections.

Glioma (brain tumor), being highly vascular, proliferative and invasive, was considered for the present study. The technique was also performed on brain cryo-sections and non-malignant brain tissue sections along with lung, kidney and thyroid tissue sections and their results were compared for reproducibility. Expression of protein human Telomerase Reverse Transcriptase (hTERT), implicated as a proliferation marker in various cancers (12-14), was used to verify and replicate the procedure, and quantitatively analyze and statistically validate the methodology using fluorescence tagged antibody. Subsequently, expression of other nuclear and cytoplasmic fluorescein isothiocyanate (FITC) tagged protein targets, namely High Mobility GroupA1 (HMGA1), Kinesin Family Member14 (KIF-14) and Synaptophysin, was also checked on glioma tissues.

\section{MATERIAL and METHODS}

\section{Treatment Reagents}

The following reagents were used in this study: Neutral detergent (Extran, Merck, UK), acetic acid (Merck, UK), ethanol (Merck, UK), poly L-lysine (Sigma, USA), sodium borohydride (Hi-media, India), crystal violet (Hi-media, India), $\mathrm{NaCl}$ (Rankem, India), sodium citrate (Qualigens, India), Phosphate buffer saline (PBS) (Thermo fisher Scientific, India), Tris (hydroxymethylaminomethaneQualigens, India), Triton X-100 (Sigma, USA), horse serum (Santa Cruz Biotechnology, USA), BSA (bovine serum albumin- Sigma, USA), hTERT primary antibody (Monoclonal, Abcam, UK), HMGA1 (Monoclonal, Abcam, UK), Synaptophysin (Monoclonal, Santa Cruz Biotechnology, USA), KIF 14 (Monoclonal, Santa Cruz Biotechnology, USA), host specific Fluorescein isothiocyanate (FITC) labeled secondary antibodies (Santa Cruz Biotechnology, USA), 4', 6-Diamidino-2-phenylindol (DAPI, Hi-Media, India), p-phenylenediamine (Anti-fade, Sigma, USA), Sudan black B (SBB, Hi-Media, India).

The specific labelling of hTERT protein in selected tissuesections by immuno-florescence immunohistochemistry (IF-IHC) was performed as per the protocol discussed here. To start with, slides were cleaned with neutral detergent, washed in running tap water for $30 \mathrm{~min}$, and further dipped in $1 \%$ acidic ethanol followed by rinsing in deionised water. The slides were then coated with poly-L-lysine solution (1:10, diluted in deionised water), then kept overnight at room temperature. $3 \mu \mathrm{m}$ sections were overlaid onto the coated slides and coded. At the beginning of the procedure, tissue sections were deparaffinized in the oven at $65^{\circ} \mathrm{C}$, followed by immersing in 3 changes of xylene, 5 minutes each. The slides were then dipped in 100\% and 95\% ethanol for 2 changes of 5 minutes each, followed by immersing in $80 \%, 70 \%$ and $50 \%$ alcohol respectively, for 5 minutes each. Finally, slides were rinsed in running tap water for 15 minutes.

\section{Pre-Treatment}

Freshly prepared $0.1 \%$ sodium borohydride in phosphate buffer saline (PBS, pH 7.4) was applied to sections for 2 changes, 2.5 minutes each without any in-between wash. Then $0.5 \%$ crystal violet ( $10 \%$ stock in methanol) freshly diluted in normal saline was laid on the sections for 9 minutes and drained. Lastly, $0.9 \% \mathrm{NaCl}$ was overlaid on the tissue, creating a bubble of liquid over the section for three washes of 5 minutes each.

\section{Epitope Retrieval}

Sodium citrate buffer $(0.01 \mathrm{M}, \mathrm{pH}=6)$ was preheated at P-high (1350 watt) for 5 minutes, in a microwave oven. The slides were immersed in this pre-warmed retrieval solution and the sections boiled for 6 minutes at P-high. After a pause of 1 minute to lower down the temperature of retrieval solution, the sections were re-heated at P-40 (540 watt) for 9 minutes. The container was removed to room temperature and the slides allowed to cool for 20 minutes. On cooling, the slides were flushed in running tap water for 15 minutes. Excess water was drained-off from the slides and the sections covered with $\mathrm{PBS}(\mathrm{pH}=7.4)$, creating a bubble of buffer over the tissue for 5 minutes.

\section{Troubleshoot}

Standardization of protocol was done by immersing the slides directly in retrieval solution and boiling the sections for 5 minutes at P-high, P-80 (1080 watt), P-60 (810 watt) and $\mathrm{P}-40$, which did not give satisfactory florescence signal intensity.

\section{Permeabilization}

The sections were permeabilized by dipping in $0.2 \%$ Triton $\mathrm{X}-100$ in PBS (pH 7.4) for 45 minutes at room temperature. Slides were rinsed and PBST ( $0.1 \%$ Tween in PBS) dropped on the tissue, creating a layer of buffer over the section for 5 minutes.

\section{Troubleshoot}

- $1 \%$ Triton X-100 in PBS (pH 7.4) for 30 minutes at room temperature

- $0.5 \%$ Triton X-100 in PBS (pH 7.4) for 45 minutes at room temperature

Both the above combinations did not yield desired results. 


\section{Blocking}

$70 \mu \mathrm{l}$ blocking buffer (10\% horse serum and $1 \%$ BSA in $\mathrm{PBS}, \mathrm{pH}=7.4$ ) was pipetted onto the sections followed by incubation in a humidified chamber at room temperature for $1 \mathrm{~h}$. Excess blocking buffer was drained off from the slides before proceeding to the next step.

\section{Troubleshoot}

- $10 \%$ horse serum and 5\% BSA in PBS, pH 7.4, at room temperature for $1 \mathrm{~h}$

- $9 \%$ horse serum and 3\% BSA in PBS, pH 7.4, at room temperature for $1 \mathrm{~h}$

- $10 \%$ horse serum and 3\% BSA in PBS, pH 7.4, at room temperature for $1 \mathrm{~h}$

- $10 \%$ horse serum and 2.5\% BSA in PBS, pH 7.4, at room temperature for $1 \mathrm{~h}$

None of the above combinations yielded complete inhibition of non-specific binding.

\section{Antigen Localization}

$70 \mu \mathrm{l}$ of primary antibody hTERT (1:750 dilutions) reconstituted in blocking buffer, was applied to the tissue sections and incubated overnight at $4^{\circ} \mathrm{C}$ in a humidified chamber. After removal from the incubation chamber, sections were covered with PBST for 5 minutes creating a bubble and then rinsed with a gentle stream of PBST (repeated thrice). Then sections were treated with $70 \mu$ l host specific FITC labelled, secondary antibody (1:300 dilution) re-constituted in PBS (pH 7.4). Sections were incubated for 60 minutes in the dark in a humidified chamber at room temperature, followed by washing as mentioned for primary antibody. Counterstaining of the sections was done using DAPI ( $5 \mu \mathrm{g} / \mathrm{mL}$ in PBS, $\mathrm{pH}$ 7.4) for 15 minutes in the dark at RT, and then the slides were washed as described in the permeabilization step. This procedure was repeated with each of the other four antibodies on glioma tissue sections. The specificity of each monoclonal antibody was checked by western blot before application.

\section{Final Treatment}

After the counterstaining procedure, sections were treated with a freshly prepared $0.1 \%$ solution of SBB in $70 \%$ ethanol, for 5 minutes in the dark. Slides were then given a final wash with PBST as per procedure followed earlier. Throughout the procedure the tissue was not touched or allowed to dry.

\section{Mounting}

Slides were mounted in the dark using antifade, placed on a filter paper to drain excess mounting medium and allowed to dry. The cover-slips were sealed with transparent nail varnish and the slides were then kept for $1 \mathrm{hr}$ at $-20^{\circ} \mathrm{C}$ for curing before visualizing.

The slides were stored in tight moisture-free boxes for 1824 months and could be reviewed thrice without fading of fluorescence signal. Each glioma sample was processed in duplicate sections for the complete procedure.

\section{Microscopy}

For epifluorescence microscopy, Axioskop 2 plus Imager (Carl Zeiss, AG, Oberkochen, Germany) equipped with a super high pressure mercury lamp (HBO 100) and VDS high resolution (1280x1024 effective pixels) cooled chargecoupled device-1300 (Hamamatsu Photonics, Hamamatsu, Japan) was used. DAPI was detected using Zeiss-01 filter set (excitation BP, 365/12 nm; beam splitter FT, 395; emission LP, $397 \mathrm{~nm}$ ) and FITC was detected using Zeiss-09 filter set (excitation BP, 450/490 nm; beam splitter FT, 510; emission LP, $515 \mathrm{~nm}$ ). All images were observed with Plan-Neofluar 40 X $0.75 \mathrm{NA}$ lens. With regard to the protein assessed, areas with highest protein labeling were considered. Approximately 1000 cells per section were randomly selected and captured with 400x magnification followed by digitalization and analysis with the Case Data Manager Expo 4.5 software (Applied Spectral Imaging, Edingen Neckarhausen, Germany). These images were exported as TIFF files to Image-J software (version 1.45, National Institute of Health, USA) for quantification of fluorescence signals of interest.

Quantification of fluorescence signals: Image-J software generated data on fluorescence signal intensity was analyzed separately by two different observers. The results were compared and found to be reproducible. The procedure adopted for this was as follows: an outline was drawn around each positively expressing cell, for calculating the area under consideration, mean fluorescence and integrated density. This step was repeated for adjacent background by creating a grid. The corrected total cellular fluorescence (CTCF) was calculated as = Integrated density - (area of selected cell $\times$ mean fluorescence of background).The value of CTCF for treated cells was then equalized against the CTCF of untreated cells in the same field of view and results compiled for the trio treatment. 


\section{Statistical Analysis}

Quantitative assessment of specifically labeled signal with increased intensity after the triple reagent treatment was compared with signals of paired untreated sections using Wilcoxon matched-pairs signed rank test (two-tailed Student's t-test; non-parametric). Results are presented as median values with inter quartile range (IQR=Q1-Q3). All analysis was done using the Graph Pad Prism software (version 7.03).

\section{RESULTS}

In this study, a preliminary screening was done to determine the efficacy of the selected agents singularly and in combination(s); and also to define the sequence that can efficiently quench autofluorescence. Treatment of copper sulphate in $50 \mathrm{mM}$ sodium acetate for 10 minutes (Figure 1A-C), ammonium chloride treatment $(50 \mathrm{mM}$ in Tris buffered saline for 10 minutes) (Figure 1B, F) and high performance UV-C light (15 W/single wavelength $254 \mathrm{~nm}$ ) treatment for 3 hours (Figure 1C-G) individually showed only negligible changes. When a combination of sodium borohydride and SBB was tried out, partial reduction of autofluorescent background and non-specific binding could be achieved (Figure 1D, H).

To validate staining integrity following our triple treatment protocol, we took a two-pronged approach: staining of the tissue specimen by conventional IF protocol (Figure 2A), and staining of section from the same block with selected reagents. All test reagent incubations were performed at RT. For all reagents, various concentrations as well as incubation periods were tested until a satisfactory result was obtained or it failed. A single application of $0.1 \%$ sodium borohydride resulted in slight dampening of autofluorescent background (Figure 2B). $0.5 \%$ crystal violet treatment (Figure 2C) resulted in partial quenching of background with maximization of both specific and nonspecific signals. Lone application of $0.1 \%$ SBB for 5 minutes (Figure 2D) eliminated background autofluorescence but also led to a drastic reduction of both specific and nonspecific signal intensities. Therefore, sodium borohydride, crystal violet and SBB, the most promising candidates, were selected for further study.

Combinational testing for treatment sequence was then performed to attain the best possible signals for protein of interest; and to achieve reproducible observations and imaging. The combination of sodium borohydride, crystal violet and SBB, in this specific order, resulted in complete dampening of autofluorescent background, masking of lipofuscin and non-specific signals; with brighter/ more intense fluorescent signal of the tagged protein (Figure 2E) as compared to these treatments alone or a combination (refer Figure 3 for CTCF values of treatments $2 \mathrm{~A}$ to $2 \mathrm{E}$ ).
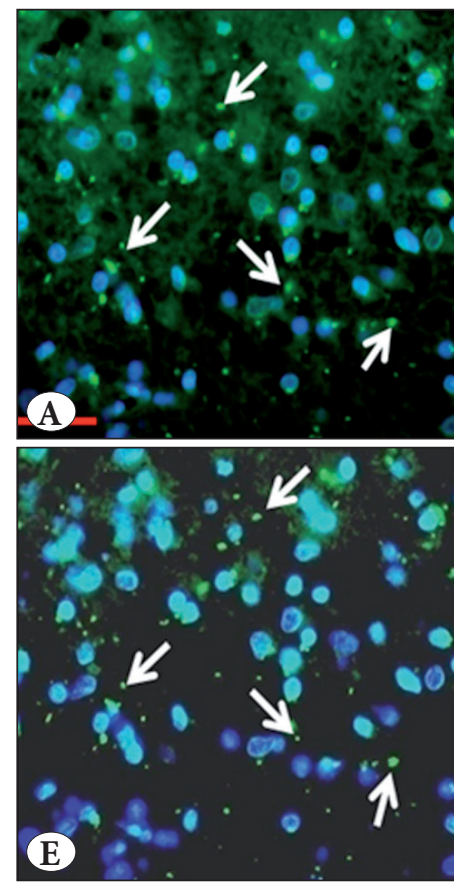
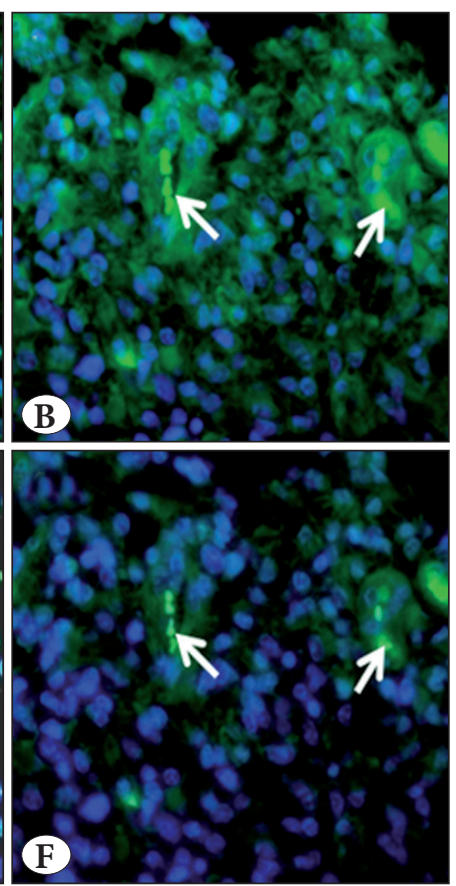
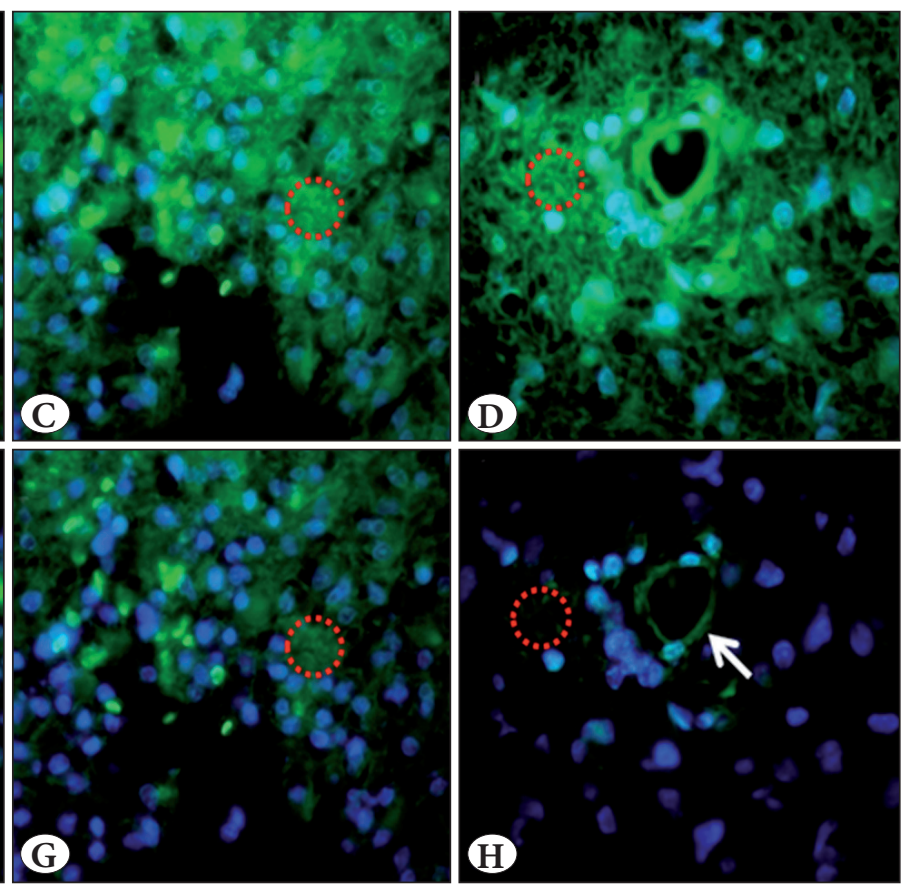

Figure 1: Superiority of present methodology over some commonly used reagents. FFPE sections A-D) processed as per conventional IF protocol. Corresponding sections treated with E) CuSO4, F) NH4Cl, G) Single wavelength $254 \mathrm{~nm}$ UV, H) Combination treatment with $\mathrm{NaBH} 4$ and SBB; all leading to only fractional reduction of autofluorescent background and non-specific binding. (Autofluorescent background is highlighted in red circle while lipofuscin and red blood cells are marked by white arrows.) (Scale Bar: $50 \mu \mathrm{m})$ 


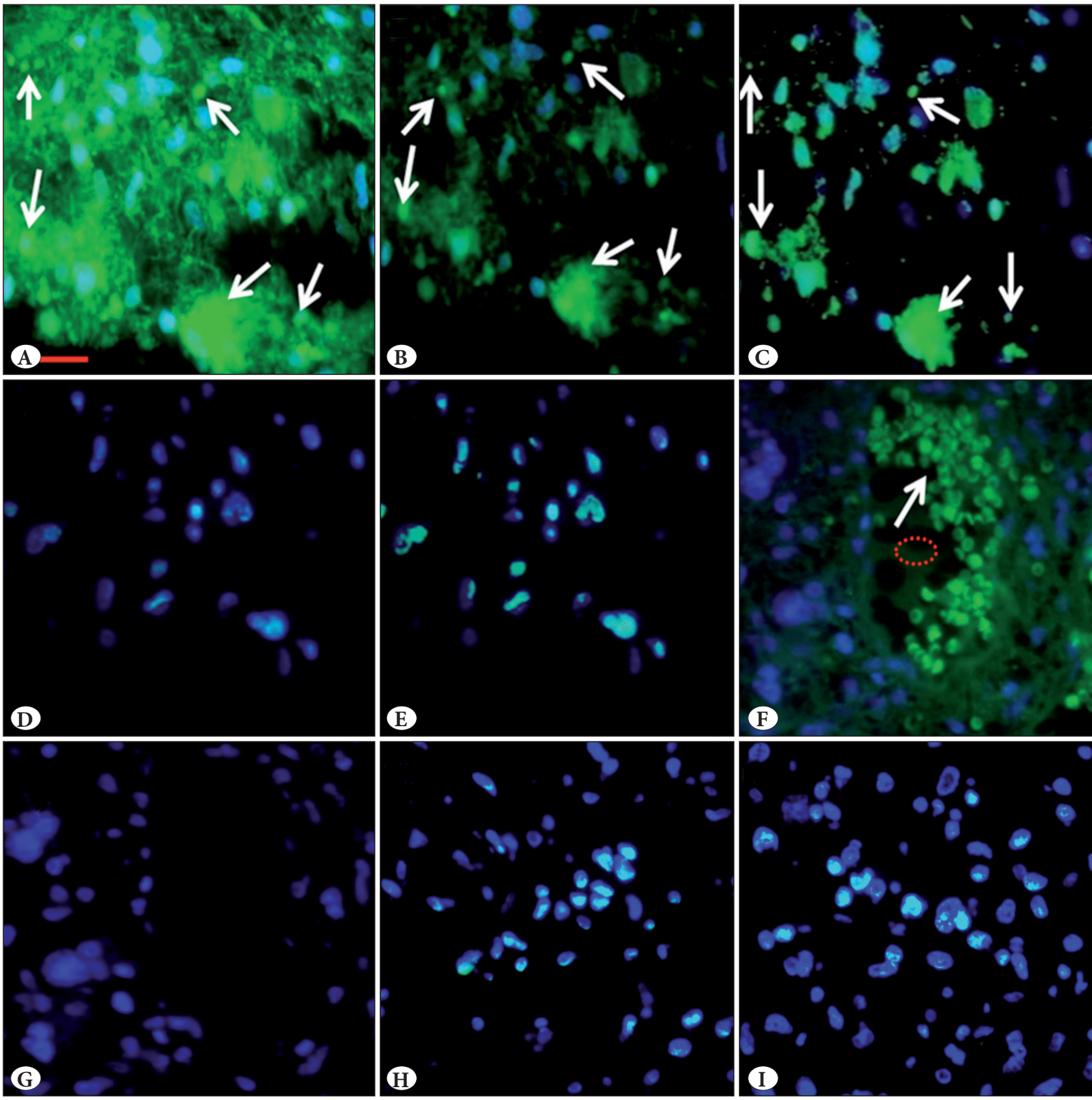

Figure 2: Histochemical treatments for reducing tissue autofluorescence. FFPE section A) Processed as per conventional IF protocol, B) Treated singularly with sodium borohydride resulting only in slight dampening of autofluorescent background, C) Crystal violet application resulted in partial quenching of autofluorescent background while maximizing both specific and non-specific signals, D) Application of SBB eliminated lipofuscin autofluorescence, resulting in total diminishing of autofluorescence but also a drastic reduction of specific and non-specific signal intensities, E) Combined treatment with sodium borohydride + crystal violet $+\mathrm{SBB}$ resulted in total quenching of autofluorescent background, masking of lipofuscin and non-specific signals. Quenching of red blood cell autofluorescence F) Red blood cells exhibiting intense green fluorescence which interferes with FITC labeled signal of tagged molecule in the section processed as per conventional IF protocol, G) Complete quenching of blood cell and background autofluorescence after trio treatment. Lipofuscin and red blood cells marked by white arrows and nonspecific green autofluorescent background highlighted with a red circle. Immunofluorescence labeled signal after storage of FFPE samples H) Image taken within 24 hours of fluorescent labeling that is on day 1, I) Section imaged again after storage at $-20^{\circ} \mathrm{C}$ for 24 months clearly indicating that the signal intensity remains the same (Scale Bar: $50 \mu \mathrm{m}$ ) 
Dealing with the other element, namely red blood cells, which exhibit green fluorescence that overlaps in the FITC channel (as seen in conventionally treated section, Figure $2 \mathrm{~F}$ ); our novel trio treatment resulted in total quenching of blood vessel autofluorescence as shown in Figure 2G. Thereafter, the order for this optimal staining combination was fixed as sodium borohydride followed by crystal violet treatment before antigen retrieval step and SBB treatment after staining with DAPI. This proved to be best suited for diminishment of autofluorescent background and nonspecific binding; paving way for successful visualization of the fluorescence tagged marker.

The increased intensity of desired signal achieved by our trio treatment has also enabled us to overcome the limitation of archiving IF labelled sections, facilitating storage for as long as 24 months with periodic review and without fading of the signal (Figure 2H, 2I; refer to Figure 3 for CTCF value). This is a huge increment on the previously reported storage period of 9 months (2). The method was also successfully assessed and validated using 3 other antibodies, with both nuclear and cytoplasmic expression (HMGA1, Synaptophysin, KIF- 14) (Figure 4A-C).

The current standardized IF-IHC protocol also has potential application on varied vascular tissue types as well as frozen brain sections, with results comparable to archival tissue (Figure 5). A negative control to check non-specific binding of secondary antibody was also employed for the fluorescence tagged molecule. No evidence of secondary antibody-induced background fluorescence (non-specific fluorescence signal) was observed for hTERT protein.

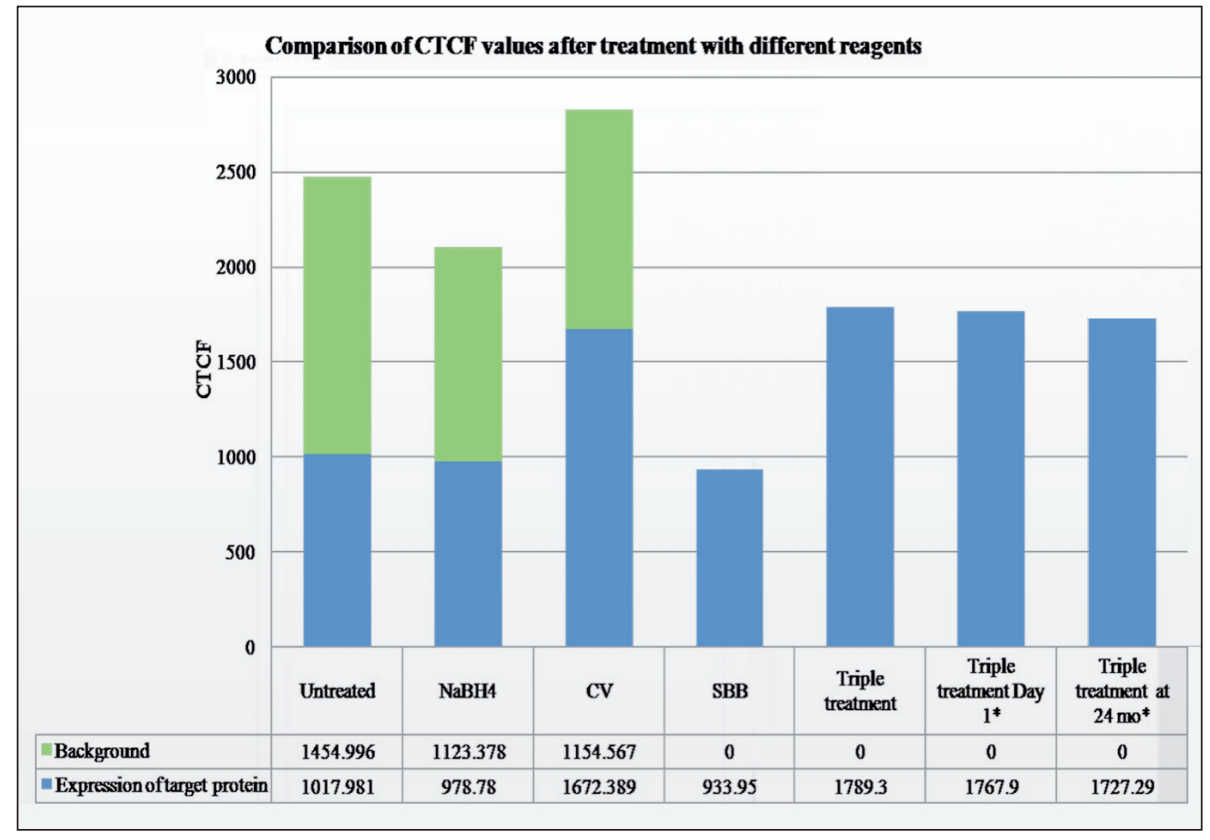

Figure 3: Graph depicts the corrected total cell fluorescence (CTCF) values of IF signals of different treatments represented in Figure $2 \mathrm{~A}$ to $\mathrm{E}$ and $2 \mathrm{H}$ to $\mathrm{I}$, indicating that trio treatment is best suited for complete quenching of autofluorescence and maximization of signal intensity of target protein.

NaBH4: Sodium-borohydride, CV: Crystal violet, SBB: Sudan Black B,

Triple treatment: Combination of $\mathrm{NaBH} 4$, $\mathrm{CV}$ and $\mathrm{SBB}$ in that order
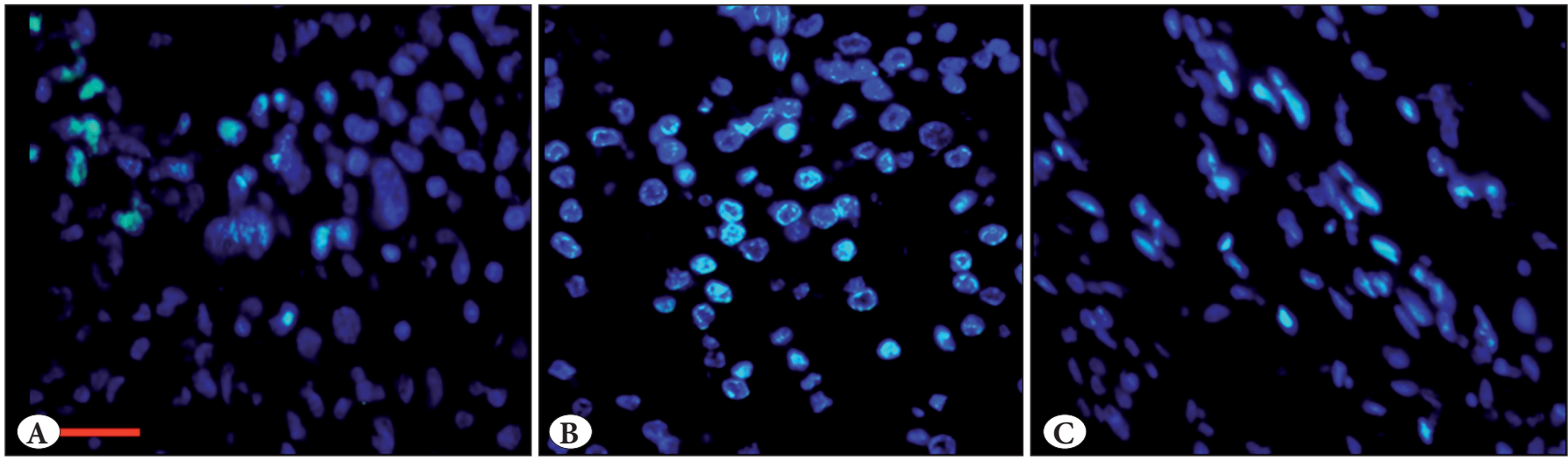

Figure 4: Nuclear and cytoplasmic immunoflorescence labeling of different primary antibodies on glioma tissue A) HMGA1, B) Synaptophysin , C) KIF-14. (Scale bar: $50 \mu \mathrm{m}$ ) 
Subsequently, the above standardized method was validated on different tissue samples and controls. The expression of hTERT was negative in non-malignant brain tissue samples; while it was up-regulated in different tumors types.

To validate the results, a quantitative analysis of 10 paired glioma tissue sections was carried out. CTCF was calculated for positive cells and background in each of these sections. The Wilcoxon signed-rank paired $t$-test showed that signal intensity of protein expressing cells in terms of CTCF of treated sections (median 3261.80 (IQR 1780.44-6075.93)) was significantly higher than CTCF of untreated sections (median 1164.54 (IQR 574.925-2225.10), $\mathrm{p}=0.002$ ). However, the signal intensity of the background in treated sections was significantly lower (median 235.174
(IQR 46.27-570.32)) than the background of untreated sections (median 2950.79 (IQR 742.71-5340.73), $\mathrm{p}=0.002$ ), validating our methodology (Table I).

\section{DISCUSSION}

IHC is a validated technique for diagnostics and research. During the use of a chromogenic system for antigen localization, problems may arise due to saturation and precipitation of substrate (15) or diffusion of the antigen (16) making quantification difficult. However, IF-IHC staining, though more sensitive, is not frequently applied to FFPE tissues in diagnostic histopathology, the perceived thought being that endogenous autofluorescence of vascular tissues renders IF imaging unreliable. In our case as well,

Table I: Corrected total cell florescence (CTCF) of untreated and treated background and cells expressing hTERT in glioma sections.

\begin{tabular}{ccccc}
\hline \multirow{2}{*}{ S.No. / per field } & \multicolumn{2}{c}{ Background } & \multicolumn{2}{c}{ Expressing Cells } \\
\cline { 2 - 5 } & Untreated & Treated & Untreated & Treated \\
\hline 1 & 5488.659 & 392.0153 & 383.272 & 2204.11 \\
\hline 2 & 4896.968 & 103.3064 & 3810.958 & 9605.009 \\
\hline 3 & 606.966 & 5.696 & 274.95 & 385.1 \\
\hline 4 & 164.634 & 18.5 & 658.43 & 1794.71 \\
\hline 5 & 10295.388 & 629.7608 & 547.09 & 888.06 \\
\hline 6 & 212.267 & 36.0638 & 1211.2 & 1775.69 \\
\hline 7 & 3816.9 & 742.35 & 2047.935 & 7924.168 \\
\hline 8 & 2084.68 & 367.0418 & 2284.164 & 4319.506 \\
\hline 9 & 10275.619 & 662.15425 & 1117.8988 & 4964.9695 \\
\hline 10 & 1149.952 & 76.9036 & 4522.887 & 6446.257 \\
\hline
\end{tabular}

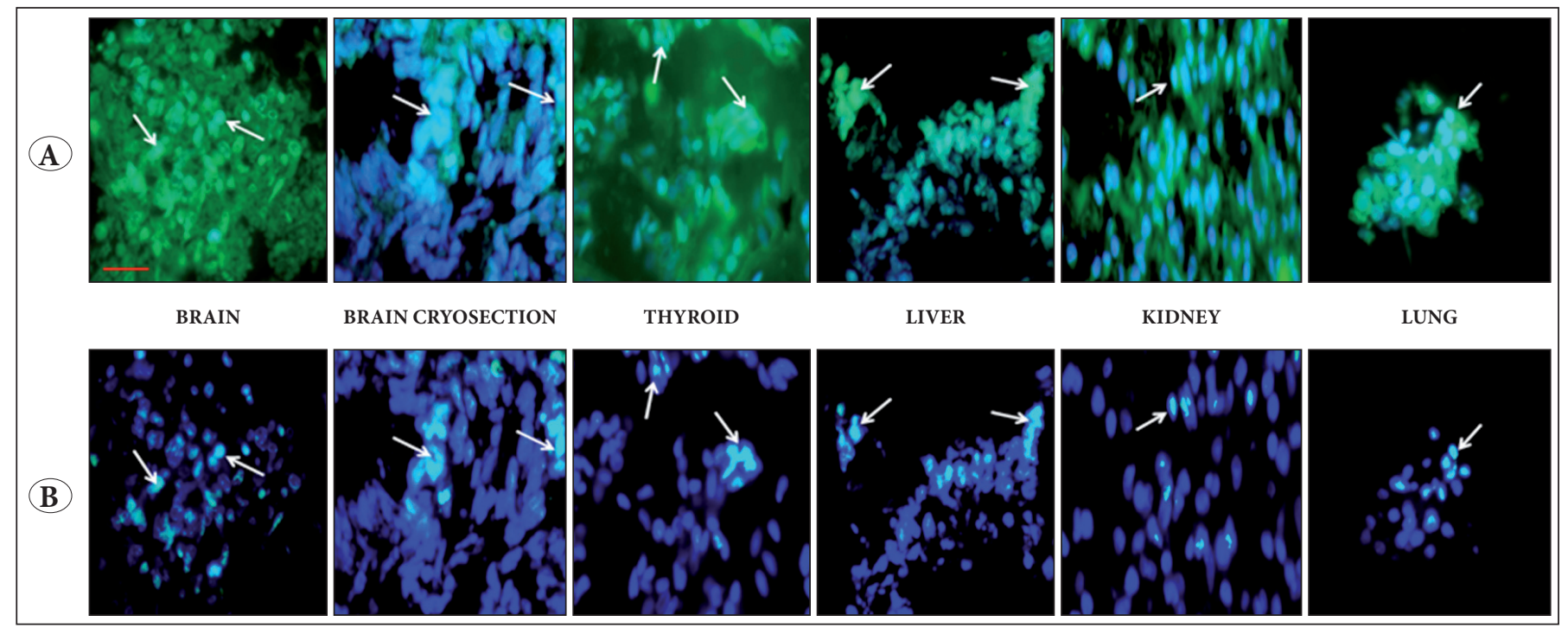

Figure 5: Expression of FITC tagged hTERT protein. A) In untreated tissue sections of brain, its cryosection, thyroid, liver, kidney and lung, B) Unambiguous hTERT expression in respective sections of different tissues after trio treatment with sodium borohydride, crystal violet and SBB. (Cells expressing the markers are indicated with white arrows, Scale Bar: $50 \mu \mathrm{m}$ ) 
efficacious visualization of specific fluorescent label was hindered by extensive autofluorescence when conventional IF protocol was employed on different human FFPE tissues and cryo sections. Therefore, the present study evaluated protocols for diminishing tissue autofluorescence in archival and cryo sections from different types of vascular tissues.

Our reagent selection was led by protocols frequently reported in the literature for quenching autofluorescence; hence sodium borohydride, crystal violet, UV-treatment, SBB, copper sulphate and ammonium chloride were selected to obtain a reduction in background. Santhosh Kumar and co-workers have recommended the use of UVlight (17). Nybo has employed ammonium chloride to quench autofluorescence (18), while Spitzeret et al. have suggested the use of copper sulphate with ammonium acetate for achieving the desired results (19).

Sodium borohydride (1\% in phosphate buffer) treatment has been used for both antigen retrieval and to reduce aldehyde induced autofluorescence of formalin fixed tissue (20). Crystal violet treatment of FFPE tissue sections is cited to reduce the endogenous autofluorescence (21) while allowing IF staining with FITC conjugated antibodies (22). Tissue treatment with $0.1 \%$ SBB in $70 \%$ ethanol has also been suggested as a good method to reduce/ eliminate tissue autofluorescence and background, while preserving the specific fluorescence signals $(23,24)$. In a study by Viegas et al. a combination of short-duration, high-intensity UV irradiation and SBB has been suggested as a better approach to reduce autofluorescence in highly vascular, high lipofuscin containing murine kidney tissues (25). Another documentation of sequential treatments by Kajimura et al. advocates the use of $0.1 \%$ sodium borohydride followed by $0.3 \% \mathrm{SBB}$ in $70 \%$ ethanol as an effective procedure for decreasing autofluorescence (26). However, the current novel trio-treatment of sodium borohydride, crystal violet and SBB gave best reproducible results in all tissues types with both nuclear and cytoplasmic antigen localization, without compromising the signal intensity on extended storage.

Our study outcome thus clearly indicates that this indirectIF based triple treatment methodology is able to provide successful quantifiable observations with a dampening of autofluorescence and non-specific background fluorescence, findings that can be extrapolated to other vascular human FFPE tissues and cryo sections for different protein targets.

\section{CONFLICT of INTEREST}

The authors declare that there are no competing interests.

\section{ACKNOWLEDGEMENTS}

The authors are thankful to M.P. Biotech Council, M.P. (project no.249) for financial assistance and BMHRC for infrastructural facilities. The present study is part of approved project IEC/21/Res/11. The authors kindly acknowledge Dr. Nitin Garg and Dr. Sandeep K Sorte, Dept. of Neurosurgery for referral of glioma cases. The authors are also thankful to Ms. Kavita Niraj for assistance in literature survey and standardization of protocol, to Dr. Hunni Gulwani and staff of Histopathology, Dept. of Pathology, for providing tissue sections.

\section{REFERENCES}

1. Schneider GEM, Straub CJ, Panzanelli P, Weinmann O, SassoèPognetto M, Fritschy JM. Immunoflorescence in brain sections: Simultaneous detection of presynaptic and postsynaptic proteins in identified neurons. Nat Protoc. 2006;1:1887-97.

2. Robertson D, Savage K, Reis-Filho JS, Isacke CM. Multiple immunofluorescence labelling of formalin-fixed paraffinembedded (FFPE) tissue. BMC Cell Biol. 2008;9:13.

3. Ramos-Vara JA, Miller MA. When tissue antigens and antibodies get along: Revisiting the technical aspects of immunohistochemistry-the red, brown, and blue technique. Vet Pathol. 2014;51:42-87.

4. Rizzardi AE, Johnson AT, Vogel RI, Pambuccian SE, Henriksen J, Skubitz AP, Metzger GJ, Schmechel SC. Quantitative comparison of immunohistochemical staining measured by digital image analysis versus pathologist visual scoring. Diagn Pathol. 2012;7:42.

5. Billinton N, Knight AW. Seeing the wood through the trees: A review of techniques for distinguishing green fluorescent protein from endogenous autofluorescence. Anal Biochem. 2001;291:17597.

6. Casella GT, Bunge MB, Wood PM. Improved immunocytochemical identification of neural, endothelial, and inflammatory cell types in paraffin-embedded injured adult rat spinal cord. J Neurosci Methods. 2004;139:1-11.

7. Croce AC, Bottiroli G. Autofluorescence spectroscopy and imaging: A tool for biomedical research and diagnosis. Eur J Histochem. 2014;58:2461.

8. Jung T, Bader N, Grune T. Lipofuscin: Formation, distribution, and metabolic consequences. Ann N Y Acad Sci. 2007;1119:97111.

9. Perse M, Injac R, Erman A. Oxidative status and lipofuscin accumulation in urothelial cells of bladder in aging mice. PLoS One. 2013;8:e59638.

10. Chen Jie, Laramore C, Shifman M. Triple-labeling whole-mount in situ hybridization method for analysis of overlapping gene expression in brain tissue with high level of autofluorescence. J Cytol Histol. 2015;S3:011. 
11. Diez-Fraile A, Van Hecke N, Guerin CJ, D'Herde K. Optimizing multiple immunostaining of neural tissue. In: Deghani $\mathrm{H}$, editor. Applications of immunocytochemistry. Rijeka: Tech Publishing;2012. 29-48.

12. Lu MH, Liao ZL, Zhao XY, Fan YH, Lin XL, Fang DC, Guo H, Yang SM. hTERT-based therapy: A universal anticancer approach (Review). Oncol Rep. 2012;28:1945-52.

13. Jun Li, Cao X, Fang Y, Liao ZE, Liu YY, Huang BD, Han YJ. Overexpression of hTERT in potentially malignant colorectal laterally spreading tumors. Mol Med Rep. 2013;7:1409-12.

14. Guan GG, Wang WB, Lei BX, Wang QL, Wu L, Fu ZM, Zhou FX, Zhou YF. UBE2D3 is a positive prognostic factor and is negatively correlated with hTERT expression in esophageal cancer. Oncol Lett. 2015;9:1567-74.

15. Glass G, Papin JA, Mandell JW. SIMPLE: A sequential immunoperoxidase labeling and erasing method. J Histochem Cytochem. 2009;57:899-905.

16. Boenisch T. Handbook on immunohistochemical staining methods. 3rd ed. Carpenteria, California, DAKO Corporation;2001. 34-8.

17. Kumar SB, Sandhyamani S, Nazeer SS, Jayasree RS. Rapid and simple method of photobleaching to reduce background autofluorescence in lung tissue sections. Indian J Biochem Biophys. 2015;52:107-10.

18. Nybo K. GFP imaging in fixed Cells. Biotechniques. 2012;52:35960 .
19. Spitzer N, Sammons GS, Price EM. Autofluorescent cells in rat brain can be convincing impostors in green fluorescent reporter studies. J Neurosci Methods. 2011;197:48-55.

20. Chen X, Cho DB, Yang PC. Double staining immunohistochemistry. N Am J Med Sci. 2010;2:241-5.

21. Buchynska L, Kashuba E, Szekely L. Immunofluorescence staining of paraffin sections: Creating DAB staining like virtual digital images using CMYK color conversion. Exp Oncol. 2008;30:327-9.

22. Kong J, Ringer DP. Scanning microfluorometric analysis of proliferating cell nuclear antigen in formalin-fixed sections of hyperplastic and neoplastic rat liver. Cytometry. 1995;20:86-93.

23. Oliveira VC, Carrara RCV, Simoes DLC, Saggioro FP, Carlotti CG, Covas DT, Neder L. Sudan Black B treatment reduces autofluorescence and improves resolution of in situ hybridization specific fluorescent signals of brain sections. Histol Histopathol. 2010;25:1017-24.

24. Sun Y, Hong Y, Zheng D, Cao Q, Wang Y, Harris D, Wang Y. Sudan Black B reduces autofluorescence in murine renal tissue. Arch Pathol Lab Med. 2011;135:1335-42.

25. Viegas MS, Martins TC, Seco F, Carmo AD. An improved and cost-effective methodology for the reduction of autofluorescence in direct immunofluorescence studies on formalin-fixed paraffinembedded tissues. Eur J Histochem. 2007;51:59-66.

26. Kanjimura J, Ito R, Manley NR, Hale LP. Optimization of singleand dual-color immunofluorescence protocols for formalin-fixed, paraffin-embesdded archival tissues. J Histochem Cytochem. 2016;64:112-24. 